\title{
Nanohybrid scaffolds with luminescent remote control
}

\author{
Anastasia V. Sochilina ${ }^{1,2, *}$, Alexander G. Savelyev ${ }^{2,3}$, Natalya V. Sholina ${ }^{3,4}$, Denis N. \\ Karimov $^{2}$, Andrey V. Nechaev ${ }^{2,5}$, Evgeny V. Khaydukov ${ }^{2,3}$, and Alla N. Generalova ${ }^{1,2}$ \\ ${ }^{1}$ Institute of Bioorganic Chemistry RAS, 117997, Moscow, Russia \\ ${ }^{2}$ Scientific Research Centre "Crystallography and Photonics" RAS, 119333, Moscow, Russia \\ ${ }^{3}$ Institute of Molecular Medicine, Sechenov First Moscow State Medical University, 119991 Moscow, \\ Russia \\ ${ }^{4}$ N.N. Blokhin Russian Cancer Research Center of Ministry of Health of the Russian Federation, \\ 115478, Moscow, Russia \\ ${ }^{5}$ Institute of Fine Chemical Technologies, Moscow Technological University, 119571, Moscow \\ Russia
}

\begin{abstract}
We report on hybrid nanocomposite scaffolds on the base of cross-linked hyaluronic acid derivative with embedded upconversion nanoparticles (UCNPs). The unique photoluminescence properties of specially designed hydrophilic UCNPs enable visualization of hydrogel using NIR irradiation. Formation of scaffold structure can be produced by means of 3D printing or direct laser writing. For the first time, we present visualization of nanohybrid scaffolds in live small animal aiming to demonstrate new possibilities of their luminescent remote control for tissue engineering.
\end{abstract}

\section{Introduction}

Hyaluronic acid (HA) appears to be one of the most suitable materials for tissue engineering and regenerative medicine. This naturally occurring polysaccharide is a priori biocompatible and biodegradable, still it lacks enough mechanical strength for constructing scaffolds. One of the methods to reinforce this polymer is photocrosslinking of HA derivatives containing double bonds. Addition of photoinitiator into hyaluronic acid glycidyl methacrylate (HAGM) water solution and irradiation it with UV/blue light makes possible crosslinking of composition resulting in solid insoluble hydrogel formation. Incorporation of UCNPs composed of $\mathrm{NaYF}_{4}$ crystals codoped with $\mathrm{Yb}^{3+}$ and $\mathrm{Er}^{3+}$ enables visualization of produced hydrogels under NIR light excitation as these UCNPs exhibit anti-Stokes luminescence under excitation at $980 \mathrm{~nm}$. Since NIR light falls in so called "transparency window" for biological tissues, it can penetrate tissue and organs up to centimetres unlike visible light.

\section{Results and discussion}

In this work double bond moieties were introduced into HA by conjugation of the polysaccharide with glycidyl methacrylate (GMA) producing derivative capable of crosslinking (HAGM). Conjugation of GMA can be carried out via reaction mechanisms of

\footnotetext{
*Corresponding author: ddraig@yandex.ru
} 
reversible transesterification or irreversible epoxy ring opening with carboxyl or hydroxyl moieties of HA [1]. In order to make core/shell $\mathrm{NaYF}_{4}: \mathrm{Yb}^{3+} / \mathrm{Er}^{3+} \mathrm{UCNPs}$ soluble in water their surface was first covered with amphiphilic SPAN-60 (sorbitan monostearate), then with polyethyleneimine for biocompatibility. HAGM (20 wt.\%) was further dissolved in water solution containing UCNPs with following addition of polyethylene glycol diacrylate (PEGDA), Mn=575 (5 wt.\%), and flavin mononucleotide (0,0004 wt.\%) as an endogenous photoinitiator [2].

The produced photocurable composition (PCC) was subjected to exposure at $365 \mathrm{~nm}$ wavelength to gelate $150 \mu \mathrm{m}$ thin hydrogel films $6 \mathrm{~mm}$ in diameter. The acquired scaffolds were implanted subcutaneously on dorsal side of mice with scaffolds without UCNPs as control. Animals were monitored with bioimaging system every day (Fig. 1, left). Intravital luminescent signal under NIR light irradiation was recorded up to $7^{\text {th }}$ day after, then strong signal was recorded post mortem from extracted scaffold. Histological analysis displayed weak inflammation reaction and slight degradation. As an example of structured scaffold we produced the structure using PEGDA-based PCC by means of direct laser writing (Fig. 1, right).
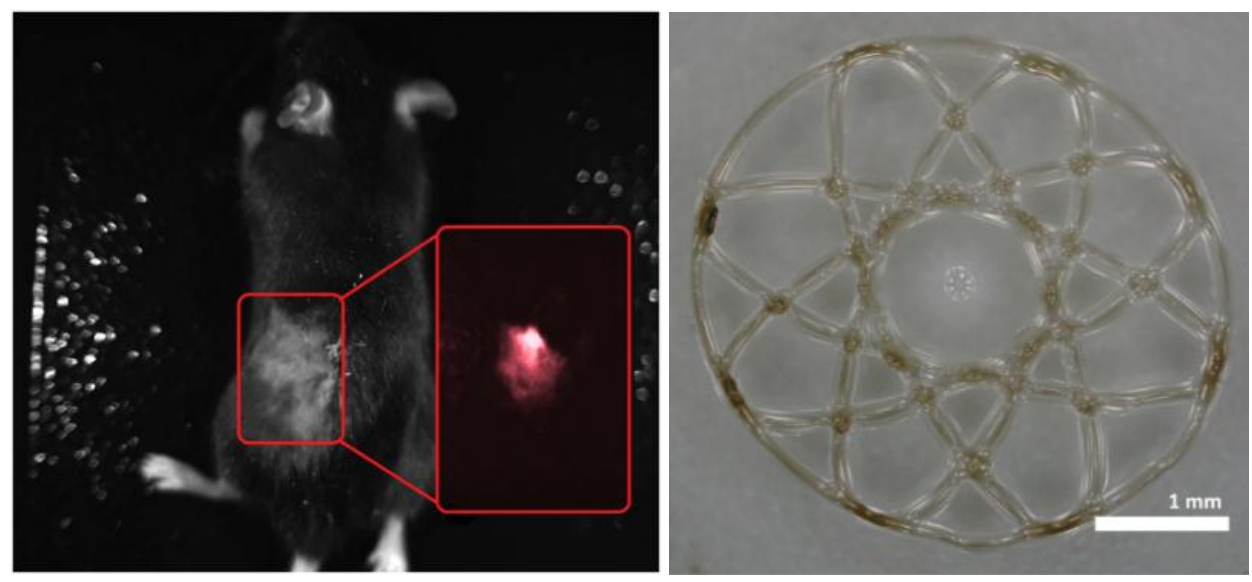

Fig. 1. Left: Bright-field photo of mouse with implanted nanohybrid scaffold on day 1. The inset shows epiluminiscent signal from scaffold under irradiation at $980 \mathrm{~nm}$ wavelength. Right: Scaffold structure produced by direct laser writing.

This scientific work has been partly supported by the Federal Agency of Scientific Organizations (Agreement No 007-ГЗ/Ч3363/26) in the part of scaffold formation and Grant RSF No. 18-79-10198 in the part of modification of UCNP surface.

\section{References}

1. S. A. Bencherif, A. Srinivasan, F. Horkay, J. O. Hollinger, K. Matyjaszewski, N. R. Washburn, Biomaterials, 29, 1739 (2008)

2. A. G. Savelyev, A. V. Sochilina, R. A. Akasov, A. V. Mironov, V. A. Semchishen, A. N. Generalova, E. V. Khaydukov, V. K. Popov, Sovremennye tehnologii v medicine, 10, 88 (2018) 